\title{
Robotic Assisted Bulbar Urethroplasty: Feasibility and Preliminar Results of the First Case-Report in Brazil
}

\author{
Marcos Tobias-Machado*, Marcio Lima and Thairo A Pereira \\ Department of Urology, Hospitais da Rede D'Or São Luiz, São Paulo, Brazil
}

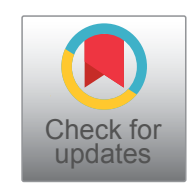

\section{Introduction}

Urethral stricture is a very challenging condition in Urology. The most common initial approach is endoscopic treatment, using incision, dilation, resection, or intralesional injection. Although, in $14-90 \%$ of the cases, there is recurrence requiring retreatment [1]. The gold standard for a durable response and long-term patency is urethroplasty, especially after a failed endoscopic treatment [2].

During the past few years, the robotic platform has rapidly increased his use in the treatment for urologic conditions, improving ergonomics and visualization not only for abdominal surgeries such as prostatectomy and cystoprostatectomy but also, for pelvic-floor disease like urethral stricture. In this article, we describe the first use of the DaVinci platform to treat a bulbar stricture successfully through pelvic access.

\section{Case Report}

A 45-years-old man, with bulbar urethral stricture due to trauma, complained about progressive obstructive symptoms. He has previously undergone an endoscopic urethrotomy, with an improvement to void at the beginning, but he returned to have symptoms 40 days after on.

The urethrogram showed a single $1.5 \mathrm{~cm}$ bulbar urethral stricture. According to that, it was indicated an urethroplasty with a direct end-to-end anastomosis. The surgeons discussed with the patient the possibility of using the platform DaVinci SI to assist the surgery. He was aware of the experimental use of the robot, and agreed with that, signing an informed consent form.

\section{Surgical Technique}

1. The patient was placed in a lithotomy position, and the operating table was leaning in a 30 degrees Trendelenburg position.

2. It was placed two trocars in the medium line of the perineum. The first one just under the scrotum, to introduce the camera, and the other one, 02 centimeters before the anus, to be used by the assistant (Figure 1).

3. Pneumoperitoneum was made, with a $15 \mathrm{mmHg}$ pressure at the beginning, which was decreased to 10

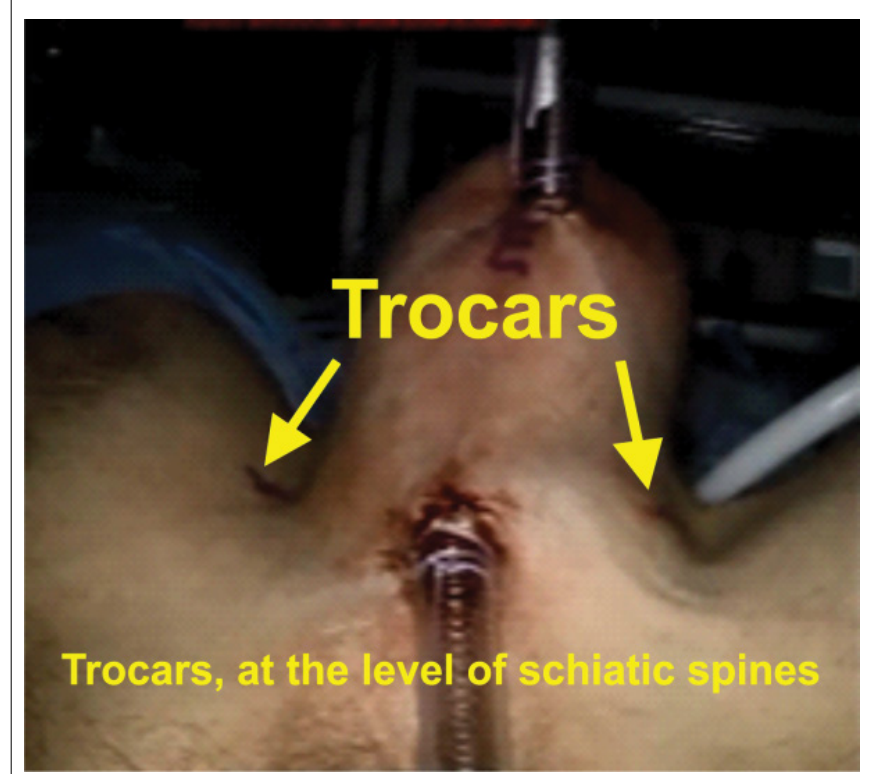

Figure 1: Trocars placement.

$\mathrm{mmHg}$ later.

4. The areolar tissue of the perineum was dissected, especially around the cavernous bodies, to allow the insertion of two more trocars, at the level of the ischiatic spines.

5. The bulbospongiosus muscle and the bulbar urethra were dissected using the bipolar grasped and the robotic scissor.

6. The stricture was identified using a cystoscopy. A guidewire was introduced through that, and a foley

*Corresponding author: Marcos Tobias-Machado, Department of Urology, Hospitais da Rede D'Or, São Paulo, Brazil

Accepted: August 25, 2021

Published online: August 27, 2021

Citation: Machado MT, Lima M, Pereira TA (2021) Robotic Assisted Bulbar Urethroplasty: Feasibility and Preliminar Results of the First Case-Report in Brazil. Adv Laparoscopy 4(1):98-101 


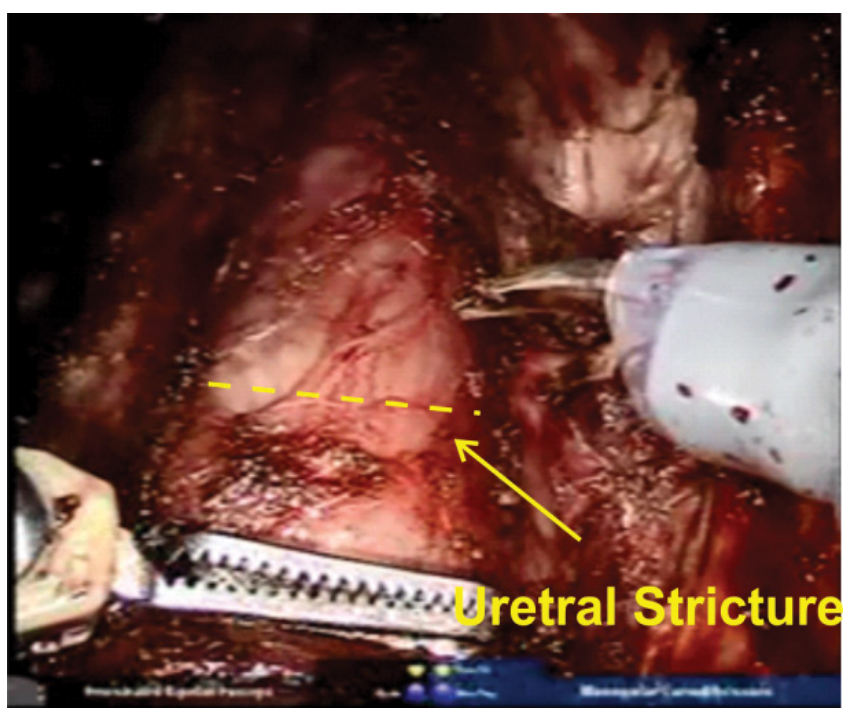

Figure 2: Stricture identified after place a Foley catheter in distal part.

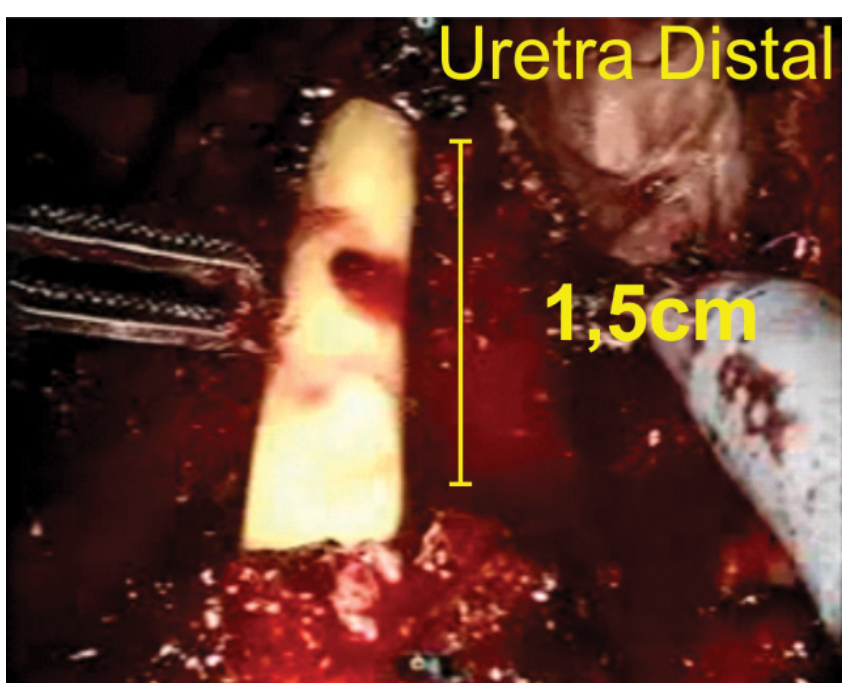

Figure 3: The stenotic segment (approximately $1.5 \mathrm{~cm}$ ) of the urethra, was sectioned.

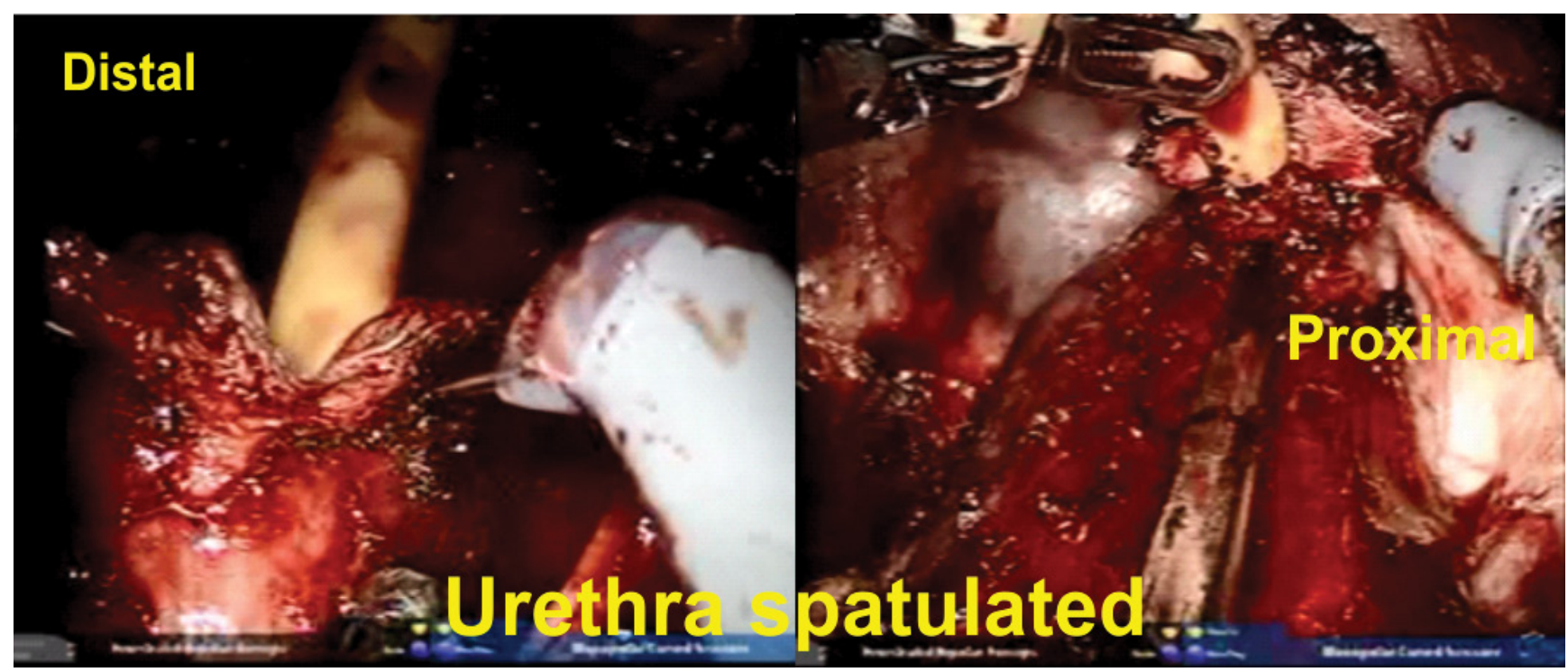

Figure 4: The urethra was spatulated, taking care to preserve the bulbar vessels.

catheter was placed in the distal part of the stricture (Figure 2).

7. The stenotic segment (approximately $1.5 \mathrm{~cm}$ ) of the urethra, was sectioned, just after it, both at the distal and proximal parts. Then, the foley catheter was placed at the bladder (Figure 3).

8. The urethra was spatulated, taking care to preserve the bulbar vessels (Figure 4).

9. The anastomosis was made with a running suture (van Velthoven technique) using a 3-0 "V-Lock" suture (Figure 5).

10. The bulbospongiosus muscle was rebuilt.

11. Placed a "Blacke" drain.

12. The subcutaneous and skin were closed with absorbable suture.

The surgery lasted 120 minutes, had very few bleeding, and had no intraoperative complications. The patient was discharged on the first post-operative day, and the foley catheter was kept for 21 days. After taking it out, the patient had no problems voiding. After two months of follow-up there, the patient is still asymptomatic.

\section{Discussion}

The utilization of the DaVinci robot significantly changed the field of Urology. Nowadays, there are many advantages of the robotic approach of the pelvis available in the literature. The male pelvic anatomy is more easily visualized; thus, the urethral anastomosis can be done with much more confidence during prostatectomy, significantly reducing the rate of bladder neck contraction [3]. Also, according to the 


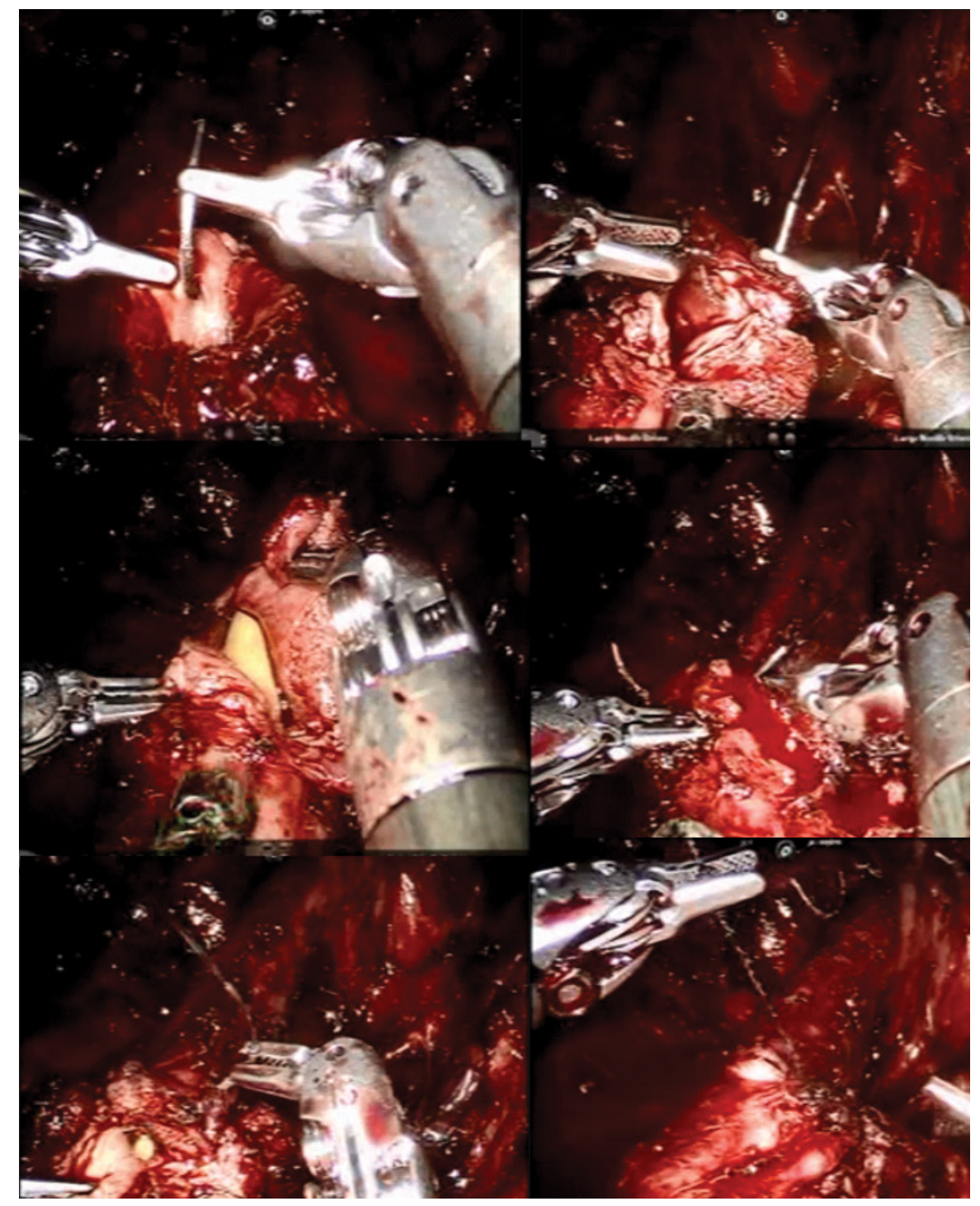

Figure 5: The anastomosis was made with a running suture (van Velthoven technique) using a 3-0 "V-Lock" suture.

Endourological Society and Society of Urologic Oncology, it has increased the ergonomic for surgeons and reduced the rate of musculoskeletal pain [4]. Unfortunately, there is no such evidence supporting the use of the robot for lower urinary tract reconstruction.

The primary goal of any treatment for urethral reconstruction is a long term patency. Depending on the etiology, the surgical technique, and the history of previous irradiation, open reconstruction reports from $60-100 \%$ of patency during follow-up. Some early robotic series reports comparable results with $75-100 \%$ of patency during a follow-up ranging from 8-24 months [5]. Although we still lack of long-term outcomes, those short term data suggest significant promise.

Approaching posterior urethral and proximal anterior strictures can be challenging because a deep perineal dissection is frequently necessary to identify and incise or excise the urethral scar tissue confined to a very narrow space limited by the lateral aspects of the pubic rami and pubic symphysis anteriorly. The space we have to perform the proximal urethral anastomosis can be extremely narrow, while the depth to reach the proximal urethra can be out of the range of vision. Despite those challenges, the robot provides vision under magnification, making it easier to preserve the irrigation of the urethra, and the gas pressures help to control bleeding.

\section{Conclusion}

Urethroplasty is the gold standard for definitive repair of urethral stricture. The DaVinci platform is a safe and promising technology to assist in the treatment, providing good visualization and approach, while improving ergonomics for the surgeon. Those advantages can ultimately simplify a complex procedure, reducing associated morbidity.

\section{References}

1. Browne BM, Vanni AJ (2017) Management of Urethral Stricture and Bladder Neck Contracture Following Primary and Salvage Treatment of Prostate Cancer. Curr Urol Rep 18: 1-9. 
2. Unterberg SH, Patel SH, Fuller TW, et al. (2019) Robotic-assisted Proximal Perineal Urethroplasty: Improving Visualization and Ergonomics. Urology 125: 230-233.

3. Webb DR, Sethi K, Gee K (2009) An analysis of the causes of bladder neck contracture after open and robot-assisted laparoscopic radical prostatectomy. BJU Int 103: 957-963.
4. Bagrodia A, Raman JD (2009) Ergonomic Considerations of Radical Prostatectomy: Physician perspective of open, laparoscopic, and robot-assisted techniques. J Endourol 23: 627-633.

5. Boswell TC, Hebert KJ, Tollefson MK, et al. (2020) Robotic urethral reconstruction: Redefining the paradigm of posterior urethroplasty. Transl Androl Urol 9: 121-131. 